\title{
Laparoscopic infrapyloric lymph nodes dissection through the right bursa omentalis approach for gastric cancer
}

\author{
Kun Yang ${ }^{1,2,3}$, Wei-Han Zhang ${ }^{1,2}$, Kai Liu ${ }^{1,2}$, Xin-Zu Chen ${ }^{1,2}$, Xiao-Long Chen ${ }^{1,2}$, Zong-Guang Zhou ${ }^{1}$ and \\ Jian-Kun $\mathrm{Hu}^{1,2^{*}}$
}

\begin{abstract}
Background: A complete dissection of infrapyloric lymph nodes is the key to a curative gastrectomy, which can be sometimes technically challenging in laparoscopic surgery.

Methods: One hundred and eighteen patients with gastric cancer undergoing laparoscopic gastrectomy with D2 lymphadenectomy in which the infrapyloric lymph nodes were dissected through the right bursa omentalis approach were included. The clinicopathologic characteristics and surgical outcomes were analyzed retrospectively.

Results: The laparoscopic gastrectomy with D2 lymphadenectomy was successful in all 118 patients with no open conversion. The mean operation time was $246.6 \pm 45.7 \mathrm{~min}$. The mean estimated blood loss was $87.0 \pm 35.9 \mathrm{~mL}$. Postoperative complications occurred in $17.8 \%$ of the patients, which were treated successfully with conservative therapy or aspiration in all. There were no No.6 lymphadenectomy-associated complications, such as injury of transverse colon, vessels of mesocolon, pancreas or duodenum, no pancreatitis, pancreatic leakage or postoperative hemorrhage. The mean postoperative hospital stay was $9.6 \pm 3.7$ days. On average, the total lymph nodes harvested were $36.8 \pm 12.9$, in which the ones from the infrapyloric area were $5.1 \pm 3.1$.
\end{abstract}

Conclusion: Laparoscopic dissection of infrapyloric lymph nodes through the right bursa omentalis approach seems to be feasible and safe, facilitating a more complete No.6 lymphadenectomy for gastric cancer.

Keywords: Gastric cancer, Laparoscopic gastrectomy, Infrapyloric lymph nodes, No.6 lymph nodes, Bursa omentalis

\section{Introduction}

The prevalence of gastric cancer has decreased worldwide in the past 20 years. However, it still remains a malignant disease with comparably high incidence and mortality especially in East Asia [1-3]. Distal gastric cancer remains the most common type, although in recent years the incidence of proximal gastric cancer has gradually increased $[4,5]$. Surgery is still the cornerstone of a

\footnotetext{
*Correspondence: hujkwch@126.com

${ }^{1}$ Department of Gastrointestinal Surgery, West China Hospital, Sichuan

University, No. 37 Guo Xue Xiang Street, Chengdu 610041, Sichuan

Province, China

Full list of author information is available at the end of the article
}

comprehensive treatment for gastric cancer in which a radical lymphadenectomy is the key [6]. Nowadays, D2 lymphadenectomy has been widely accepted as a standard procedure since it has been proven effective to reduce the risk of tumor recurrence and improve the overall survival of patients with gastric cancer [7].

The No.6 lymph nodes are defined as the infrapyloric lymph nodes along the first branch and proximal part of the right gastroepiploic artery down to the confluence of the right gastroepiploic vein and the anterior superior pancreatoduodenal vein [8]. The No.6 lymph nodes have been reported of high frequency of metastasis in gastric cancer (up to approximately $40 \%$ in advanced cases, [9] 
especially the tumor in the distal $1 / 3$ of the stomach). In the Japanese Gastric Cancer Treatment Guideline, it is recommended that the dissection of No.6 lymph nodes needs to be complete, even in the extent of D1 lymphadenectomy for distal and total gastrectomy [6]. Therefore, a thorough and precise dissection of the No.6 lymph nodes is critical.

Laparoscopic gastrectomy, the minimal invasive approach, has been increasingly used to treat gastric cancer. In the Japanese Gastric Cancer Treatment Guidelines, laparoscopic distal gastrectomy has been recommended as a treatment option for early gastric cancers [6]. Meanwhile, laparoscopic distal gastrectomy for advanced cancers and laparoscopic total gastrectomy have also been reported of comparable short-term and long-term outcomes to open surgery [10-13]. Nevertheless, accomplishing a complete and safe laparoscopic dissection of the No.6 lymph nodes is sometimes technically challenging, due to not only an intricate network and multiple anatomical variations of blood vessels in the infrapyloric area, but also the adjacent organs such as pancreas and transverse mesocolon which increase the technical difficulty and are vulnerable to potential intraprocedural injury [14]. Therefore, defining a proper surgical dissection plane with an appropriate technique is essential for a successful laparoscopic dissection of No.6 lymph nodes.

In order to facilitate a complete and safe lymphadenectomy, we have proposed a model called "clockwise modularized lymphadenectomy" for laparoscopic dissection of lymph nodes, and compared it with traditional laparoscopic lymphadenectomy [15]. In this article, we aimed to describe our surgical technique in detail for laparoscopic dissection of the infrapyloric lymph nodes through the right bursa omentalis approach, which is the important component of clockwise modularized lymphadenectomy, in order to cue a complete and safe laparoscopic No.6 lymphadenectomy and facilitate a wider application of this procedure. Additionally, we also sought to present the short-term outcomes of the procedure.

\section{Materials and methods}

\section{Patients and indications}

From January 2015 to July 2017, one hundred and eighteen patients with gastric cancer undergoing laparoscopic gastrectomy with D2 lymphadenectomy at West China Hospital, Sichuan University were included. Informed consent for operation was obtained preoperatively from all patients. The clinicopathologic characteristics and surgical outcomes, including demographic data, estimated blood loss, the number of harvested lymph nodes, TNM stage, perioperative and postoperative complications etc. were extracted from the prospective Surgical Gastric
Cancer Patient Registry in West China Hospital. The application of data from this database has been approved by the Biomedical Ethics Committee of the West China Hospital, Sichuan University which also waived the patient informed consent due to the retrospective nature of the study [IRB No. 2014(215)]. All patients' data were collected and analyzed anonymously.

All the patients included had been diagnosed of gastric cancer based on upper endoscopy and biopsy. And the preoperative contrasted abdominopelvic computed tomography was used for clinical staging. The indications for laparoscopic gastrectomy (together with curative D2 lymphadenectomy) were based on the Chinese laparoscopic gastrectomy guideline for gastric cancer (2016 edition), expert consensus on quality control of the laparoscopic radical resection for gastric cancer in China (2017 edition) and the inclusion and exclusion criteria of the CLASS-01 trials launched by the Chinese Laparoscopic Gastrointestinal Surgery Study (CLASS) group, $[10,16,17]$ where the cancer stage was defined as cT1$3 \mathrm{~N} 0-2 \mathrm{M} 0$ at preoperative evaluation according to the 7th AJCC Cancer Staging [18] Additionally, the cT4a stage was considered as an explorative indication, should the expected curative D2 lymphadenectomy be achieved. Exclusion criteria were previous upper abdominal surgery (except laparoscopic cholecystectomy), eligibility for endoscopic treatments, and enlarged regional lymph node with the diameter larger than $3 \mathrm{~cm}$.

\section{Surgical techniques}

In the present study, we aimed to describe the novel surgical approach developed for infrapyloric lymph nodes dissection that has been gradually applied on all patients undergoing laparoscopic gastrectomy since 2014 at our institution. The aim of this delicate new approach was to reduce the iatrogenic injury of blood vessels and adjacent organs in a loose natural surgical plane, and eventually achieve a technically easier, safer and complete infrapyloric lymph nodes (No.6) dissection, rather than improve the survival through bursectomy. All the operations were performed by Jian-Kun Hu (operator), Kun Yang (assistant) and Xin-Zu Chen (assistant), who are highly experienced in this technique. The operation team has performed annually more than 200 gastrectomies with D2 lymphadenectomy with open and laparoscopic approaches and is certified by the CLASS academic committee (unedited operation Additional file 1: Video S1).

Under general anesthesia, the patient was placed in the supine position. The surgeon and camera holder stood on the patient's right side and an assistant surgeon was on the patient's left side. One $12 \mathrm{~mm}$ Trocar was placed just under the umbilicus to keep the pneumoperitoneal pressure at $12 \sim 14 \mathrm{~mm} \mathrm{Hg}$ and function as the observation 
port. One $5 \mathrm{~mm}$ trocar was placed on the right anterioraxillary line just below the costal margin, and another $12-\mathrm{mm}$ port was placed on the right mid-clavicular line 2-3 $\mathrm{cm}$ above the umbilicus. Two $5 \mathrm{~mm}$ trocars were placed on left anterior-axillary line below the costal margin and left mid-clavicular line $2-3 \mathrm{~cm}$ above the umbilicus respectively.

The surgery began with liver retraction utilizing a purse-string suture. After the left-sided omentectomy and No.4sb lymph nodes dissection, the right-sided omentectomy was performed toward the hepatic flexure of colon. A "three point technique" was adopted constantly to expose the interfascial space between the inner layer and external layer of omental bursa. Ultrasonic scalpel was used for the dissection. The operator's left hand and assistant's right hand retracted and expanded the greater omentum (or anterior layer of transverse mesocolon), and the assistant's left hand pulled the transverse colon caudally and dorsally. By retracting at 3 points, 2 planes were created by dissecting the loose interfascial space between the inner layer and external layer of the omental bursa. Thereafter the operator used the ultrasonic scalpel to dissect along the border shared between the two planes to separate the anterior layer of mesocolon (Fig. 1).

The operator's left hand and the assistant's right hand retracted and expanded the greater omentum, and the assistant's left hand pulled the transverse colon caudally and dorsally and flattened the transverse mesocolon. The fourth layer of greater omentum was cut from the avascular area of the median part of gastrocolic ligament close to the transverse colon. Along this plane,

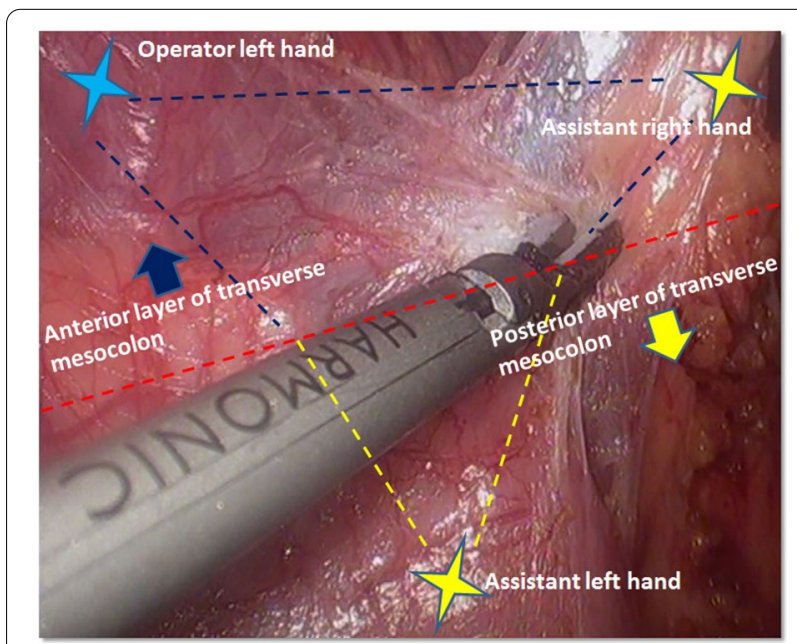

Fig. 1 A "three-point technique" was adopted constantly to expose the interfascial space between the inner layer and external layer of omental bursa the anterior layer of mesocolon peritoneum should be removed up to hepatic flexure and inferior border of pancreas cranially.

Since the interfascial space between the anterior layer and posterior layer of the mesocolon peritoneum is loose, a combination of blunt and sharp dissections can be adopted. The posterior layer can be pushed caudally with the ultrasonic scalpel. Blunt dissection of the anterior layer is also safe and very useful. When the anterior layer of transverse mesocolon peritoneum was dissected up to the inferior border of the pancreas, the surgical plane was re-oriented to the anterior surface of the pancreas. The ligaments between the duodenum and pancreas were dissected and the gastroduodenal artery was exposed, while the capsule of pancreas did not need to be peeled.

For a complete No. 6 lymph nodes dissection, the groove between the head of pancreas and the transverse mesocolon should be exposed (Fig. 2a). Then, all the fatty and lymphatic tissues in front of the surface of the pancreatic head should be completely removed upward from the groove to duodenal bulb and leftward to the descending part of the duodenum. After dissecting this area, a continuous membrane extending from the posterior layer of transverse mesocolon to the pancreatic head can be seen. During the process, the confluence of anterior superior pancreaticoduodenal vein to the right gastroepiploic vein was displayed. And the right gastroepiploic vein should be ligated and divided distal to the confluence point between the right gastroepiploic vein and anterior superior pancreaticoduodenal vein (Fig. 2b). Afterwards, the No.6a lymph nodes surrounding the right gastroepiploic artery were dissected and the right gastroepiploic artery just distal to the branching point of anterior superior pancreaticoduodenal artery from gastroduodenal artery was divided (Fig. 2c). Attention was paid to the nearby infrapyloric artery that also needed to be ligated to remove the No.6i lymph nodes. Finally, the inferior wall of the duodenal bulb was skeletonized. Thus, the infrapyloric lymph nodes were removed en bloc with the stomach. Routine dissection of No.14v lymph nodes was unnecessary, unless metastatic No.6 lymph nodes were intraoperatively suspected. The operators need to avoid injuring the pancreas, especially when there was a tongue papillae of pancreas in this area, as well as the duodenum when approaching it.

\section{Statistical analysis}

The SPSS 19.0 statistics software (SPSS Inc., Chicago, IL) was used to conduct all statistical analyses. Continuous data were expressed as mean \pm standard deviation, and categorical variables were expressed as number (\%). Statistical significance was defined as two-sided $\mathrm{p}<0.05$. 

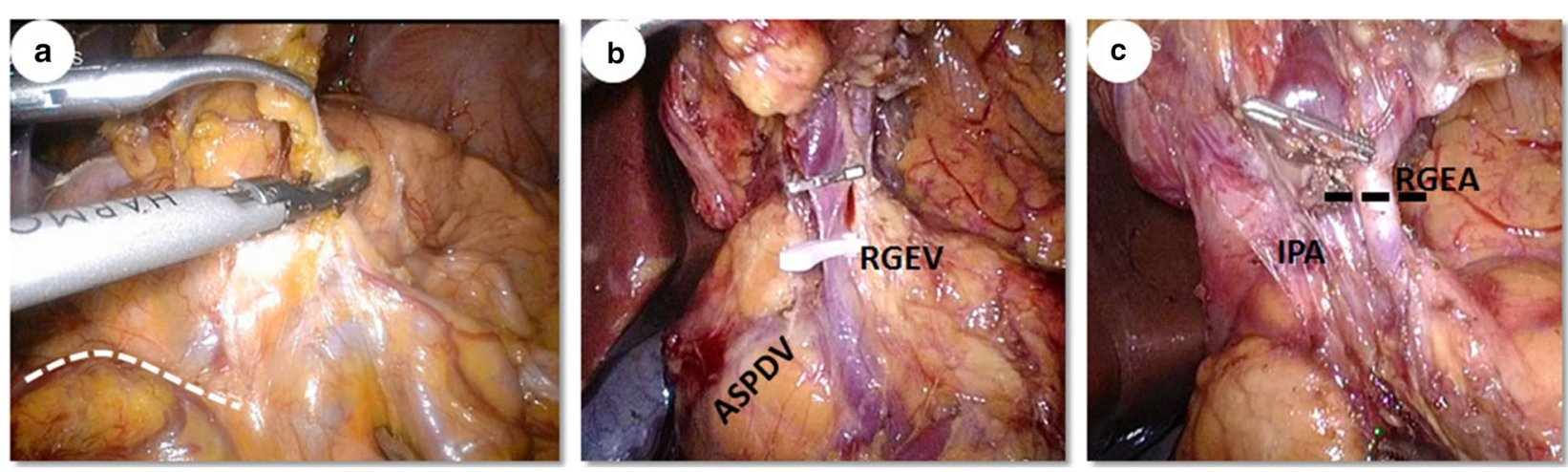

Fig. 2 Laparoscopic infrapyloric lymph nodes dissection through the right bursa omentalis approach. a The groove between the head of pancreas and the transverse mesocolon should be exposed (indicated by the broken line). $\mathbf{b}$ The right gastroepiploic vein (RGEV) should be ligated and divided distal to the confluence point between the right gastroepiploic vein and anterior superior pancreaticoduodenal vein (ASPDV). c The right gastroepiploic artery (RGEA) should be divided just distal to the branching point of anterior superior pancreaticoduodenal artery from gastroduodenal artery (indicated by the broken line). IPA infrapyloric artery

\section{Results}

Totally, one hundred and eighteen patients (92 male, $78 \%$ ) were included in analysis. The mean age was $55.4 \pm 10.7$ years and the body mass index was $22.2 \pm 2.8 \mathrm{~kg} / \mathrm{m}^{2}$. The tumor located in 74 patients at the lower $1 / 3$ of the stomach, 11 at middle $1 / 3$ and 33 at upper $1 / 3$. The patients' characteristics are summarized in Table 1.

The laparoscopic gastrectomy with D2 lymphadenectomy was successful without open conversion in all patients. Thirty five patients underwent total gastrectomy and 83 patients distal gastrectomy. The mean operation time was $246.6 \pm 45.7 \mathrm{~min}$, where the mean time for No.6 lymph nodes dissection was $32.1 \pm 5.8 \mathrm{~min}$. The mean estimated blood loss was $87.0 \pm 35.9 \mathrm{~mL}$.

There was no peri-operative mortality. Postoperative complications happened in 21 patients (17.8\%), including pulmonary infections or plural effusion in 15, intraperitoneal abscesses in 3, surgical site infections in one, and gastroplegia in 2. All complications were successfully treated with conservative therapy or aspiration. There was no No.6 lymphadenectomy-associated complications, such as injury of transverse colon, blood vessels of mesocolon, pancreas or duodenum, pancreatitis, pancreatic leakage and postoperative hemorrhage. The mean time to first flatus was $4.9 \pm 1.3$ days, mean time to first food intake $3.3 \pm 1.1$ days and mean hospital stay $9.6 \pm 3.7$ days.

The mean tumor size was $3.0 \pm 1.4 \mathrm{~cm}$. The mean number of total lymph nodes harvested was $36.8 \pm 12.9$ and No.6 lymph nodes retrieved $5.1 \pm 3.1$, where the mean number of overall metastatic lymph nodes was $1.8 \pm 2.9$ and metastatic No.6 lymph nodes $0.2 \pm 0.6$. No.6 lymph nodes metastases were observed in 15 patients. There were 35 patients in stage Ia, 22 stage Ib, 23 stage IIa, 13 stage IIb, 16 stage IIIa, 6 stage IIIb and 3 stage IIIc according to the 7th AJCC Cancer Staging.

\section{Discussion}

In the present study, we showed satisfactory short term outcomes and dissecting efficacy of laparoscopic infrapyloric lymphadenectomy for gastric cancer. Compared with our 53 patients receiving traditional procedure going inside the omental sac and dissecting the No.6 lymph nodes from the lower edge of pancreas toward to the duodenum, the novel approach retrieved more total lymph nodes $(36.8 \pm 12.9$ vs. $31.8 \pm 10.5, \mathrm{p}=0.009)$ and No.6 lymph nodes $(5.1 \pm 3.1$ vs. $3.7 \pm 2.1, \mathrm{p}=0.002)$. Meanwhile, the operation time $(246.6 \pm 45.7 \mathrm{~min}$ vs. $261.5 \pm 52.6 \mathrm{~min}, \mathrm{p}=0.078$ ), volume of blood loss $(87.0 \pm 35.9 \mathrm{~mL}$ vs. $93.8 \pm 30.8 \mathrm{~mL}, \mathrm{p}=0.212)$, and postoperative complication rates $(17.8 \%$ vs. $22.6 \%, \mathrm{p}=0.458)$ were comparable. No No.6 lymphadenectomy-associated complications were observed. Lastly, the mean number of total lymph nodes harvested and No.6 lymph nodes retrieved was comparable, or even higher than those of laparoscopic or open surgery reported in previous literatures [14, 19-23].

Lymphadenectomy is an important component of gastric cancer surgery, and D2 lymphadenectomy has been proven to decrease the locoregional recurrence and gastric cancer related deaths nowadays [7]. Because No.6 lymph nodes is one of the most frequently involved stations, complete No.6 lymph nodes dissection is crucial for a curative gastrectomy even in a D1 or D1+lymphadenectomy for early cancer, let alone advanced cancer [6, 14]. No.6 lymph nodes mainly drain the lymphatic flow from the lower $1 / 3$ 
Table 1 Clinicopathologic characteristics, operative and pathological results of the patients

\begin{tabular}{|c|c|}
\hline Variables & $\mathrm{N}=118$ [Median, Range] \\
\hline \multicolumn{2}{|l|}{ Demographics } \\
\hline Male/female & $92(78.0 \%) / 26(22.0 \%)$ \\
\hline Age (years) & $55.4 \pm 10.7[30-77]$ \\
\hline Body mass index $\left(\mathrm{kg} / \mathrm{m}^{2}\right)$ & $22.2 \pm 2.8[22.1,16-30.4]$ \\
\hline Tumor location (Upper/Middle/Lower) & $33(28.0 \%) / 11(9.3 \%) / 74$ (62.7\%) \\
\hline \multicolumn{2}{|l|}{ Operative results } \\
\hline Gastrectomy (Total/distal) & $35(29.7 \%) / 83(70.3 \%)$ \\
\hline Operative time (minutes) & $246.6 \pm 45.7[240,175-440]$ \\
\hline Time for No.6 lymphadenectomy (minutes) & $32.1 \pm 5.8[25-42]$ \\
\hline Blood loss (mL) & $87.0 \pm 35.9[20-230]$ \\
\hline Open conversion & 0 \\
\hline \multicolumn{2}{|l|}{ Postoperative recovery } \\
\hline Mortality & 0 \\
\hline Complications & $21(17.8 \%)$ \\
\hline Pulmonary infections or plural effusion & $15(12.7 \%)$ \\
\hline Intraperitoneal abscesses & $3(2.5 \%)$ \\
\hline Surgical site infections & $1(0.8 \%)$ \\
\hline Gastroplegia & $2(1.7 \%)$ \\
\hline First flatus (days) & $4.9 \pm 1.3[2-8]$ \\
\hline First liquid diet intake (days) & $3.3 \pm 1.1[2-6]$ \\
\hline Postoperative hospital stay (days) & $9.6 \pm 3.7[6-28]$ \\
\hline \multicolumn{2}{|l|}{ Pathologic results } \\
\hline Tumor size $(\mathrm{cm})$ & $3.0 \pm 1.4[3,0.5-8]$ \\
\hline No. of total retrieved lymph nodes & $36.8 \pm 12.9[12-79]$ \\
\hline No. of retrieved No.6 lymph nodes & $5.1 \pm 3.1[0-17]$ \\
\hline Mean number of overall metastatic lymph nodes & $1.8 \pm 2.9[0-14]$ \\
\hline Mean number of No.6 metastatic lymph nodes & $0.2 \pm 0.6[0-3]$ \\
\hline Patients with No.6 lymph nodes metastasis & $15(12.7 \%)$ \\
\hline $\operatorname{Stage}^{*}|/||/|||$ & $57(48.3 \%) / 36(30.5 \%) / 25(21.2 \%)$ \\
\hline
\end{tabular}

Data are mean \pm standard deviation or $\mathrm{n}(\%)$

*According to the 7th edition Cancer Staging, American Joint Committee on Cancer

of the stomach which remains the most common site of gastric cancer although the incidence of proximal tumor has been increasing in the past two decades [5, 24]. Therefore, the metastatic rate of No.6 lymph nodes is quite high. Our previous study found that the metastatic rate of No.6 lymph nodes was $28.1 \%$, [14] and another study has reported that the No.6 lymph nodes were the most frequently affected lymph nodes with the positive rate of $34.3 \%$ among patients with distal gastric cancer [25]. Consequently, the likelihood of residual disease in infrapyloric lymph nodes might increase for gastric cancer patients receiving incomplete No.6 lymphadenectomy. For them, actually, the positive No.6 lymph nodes might not be removed completely. Nevertheless, a complete No.6 lymph nodes dissection during laparoscopic surgery should not be regarded as a technically easy procedure.
Bursectomy has been proposed by Japanese surgeons since 1960s with the purpose to eliminate the possible invisible tumor seeding in the lesser sac of peritoneal cavity and completely remove the infrapyloric lymph nodes [26, 27]. Unfortunately, bursectomy has not been demonstrated to provide better survival outcome for resectable gastric cancer than non-bursectomy in a multicenter randomized controlled trial [28]. However, bursectomy could yield more retrieved lymph nodes than non-bursectomy; [29] and Blouhos et al. considered that an easy and complete en bloc infrapyloric lymph nodes dissection could be achieved through the surgical plane of right-sided bursectomy [27]. Although retrieving more lymph nodes does not necessarily associate with better long-term clinical outcomes, the removal of more lymph nodes improves the chance of cure theoretically and decreases the possibility of 
stage migration. In the present study, we also showed that laparoscopic infrapyloric lymph nodes dissection through the right bursa omentalis approach could contribute defining the proper surgical plane, which could minimize potential free cancer cell leakage caused by the transection of lymphatic vessels in an inappropriate plane, $[30,31]$ and facilitate an easy and complete lymphadenectomy. Although bursectomy is a technically challenging procedure especially performed with laparoscopy, our previous study showed that it could be performed safely by experienced surgeons [29]. Also, no bursectomy-associated complications were observed in the present study. The major risks of laparoscopic No.6 lymph nodes dissection through the right bursa omentalis approach are potential iatrogenic injury of colonic vessels or pancreatic parenchyma. Therefore, a thorough understanding of the anatomy of this area is critical. Identification of important anatomic structures, including the marginal vessel of the transverse colon, the middle colic vessels, inferior border of the pancreas, is crucial in order to avoid iatrogenic injury and recognize the correct surgical planes. Additionally, the interfascial space is generally loose on the right aspect of the transverse mesocolon. Thus, it is easier to perform the right-sided bursectomy only.

Our approach provides potential benefits for laparoscopic No.6 lymphadenectomy. Firstly, our procedure could provide a more complete infrapyloric lymph nodes dissection and avoid omission of lymph nodes, such as the right part of No.6v lymph nodes located near to the duodenum and the lymph nodes clinging to the pancreatic capsule, which is prone to be ignored when dissected in laparoscopic manner. Secondly, we indeed found lymph nodes locating just below the anterior superior pancreatoduodenal vein (Fig. 3), although the frequency was not high. Even though these lymph nodes should not be recognized as No.6 lymph nodes according to the Japanese Gastric Cancer Classification, [8] we believe that these lymph nodes have significant lymphatic channel that drains in the infrapyloric region, and communicate with the No.6 lymph nodes broadly. Therefore, we advocate to dissect these lymph nodes simultaneously and the right bursa omentalis approach is helpful to identify and dissect them. In addition, our procedure is also beneficial to expose the anterior superior pancreatoduodenal vein and the confluence of anterior superior pancreaticoduodenal vein to the right gastroepiploic vein. Finally, this procedure facilities defining the correct surgical plane and removing the whole greater omentum, which is usually integrated in the standard gastrectomy for T3 or deeper tumors [6]. A wrong plane might cause the

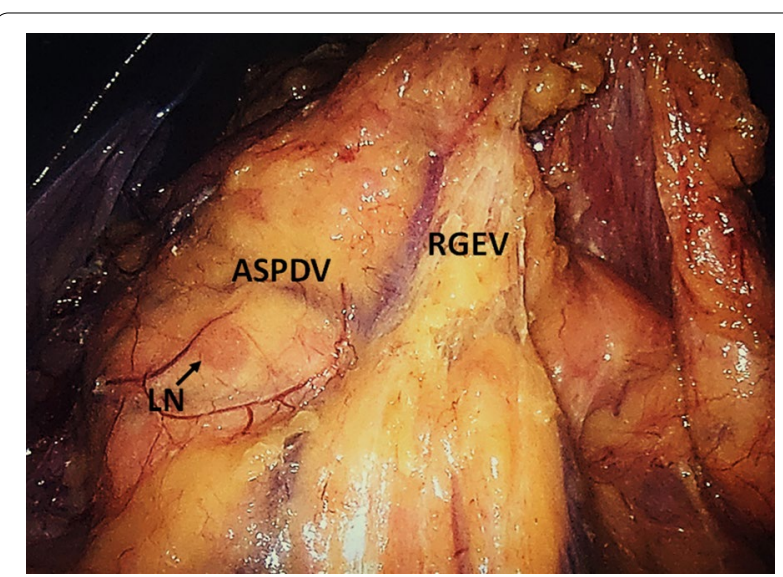

Fig. 3 Lymph nodes (LN) located just below to the anterior superior pancreatoduodenal vein (ASPDV). $R G E V$ right gastroepiploic vein

residual of the greater omentum and residual No.6 lymph nodes at the side of the transverse mesocolon.

This study has a few limitations. Firstly, this study is a retrospective, single arm study. Nevertheless, as discussed above the current approach demonstrated comparable or better results compared with traditional procedure. Secondly, some patients with early stages had been included in the study. From our point of view, the loosed natural surgical plane is helpful to reduce the iatrogenic injury of blood vessels and adjacent organs, which facilitated an easier, safer and more complete infrapyloric lymph nodes dissection. It was reported that an easy and complete en bloc infrapyloric lymph nodes dissection could be achieved through the surgical plane of right-sided bursectomy [27]. However, one should notice that for patients with early stages, our procedure was a comparably more aggressive surgical approach, and cautions need to be warranted when performing the procedure on patients with early stages. Despite these limitations, the purpose of this study was to describe the laparoscopic infrapyloric lymph nodes dissection through the right bursa omentalis approach in detail and to present data suggest the safety and feasibility of this procedure. To our knowledge, this is the first study to describe a detailed procedure for performing laparoscopic infrapyloric lymph nodes dissection through the right bursa omentalis approach for gastric cancer.

\section{Conclusions}

Laparoscopic infrapyloric lymph nodes dissection through the right bursa omentalis approach seems to be feasible and safe, facilitating a more complete No.6 lymphadenectomy for gastric cancer. The positive results need to be confirmed with prospective studies. 


\section{Supplementary Information}

The online version contains supplementary material available at https://doi. org/10.1186/s12893-021-01192-5.

Additional file 1: Video S1. This operation video shows the surgical technique of laparoscopic infrapyloric lymph nodes dissection through the right bursa omentalis approach.

\section{Acknowledgements}

This work was internal supported by Volunteer Team of Gastric Cancer Surgery (VOLTGA), West China Hospital, Sichuan University, P.R.China. The authors appreciated Galiullin Danil (Department of Gastrointestinal Surgery, West China Hospital, Sichuan University) and Claire Ren (Department of Interventional Cardiology, Erasmus Medical Center, Rotterdam, The Netherlands) for modifying the language of manuscript.

\section{Authors' contributions}

Conception or design of the work: YK, ZZG, HJK. Acquisition, analysis, or interpretation of data for the work: YK, ZWH, LK, CXZ, CXL, ZZG, HJK. Drafting the work or revising it critically for important intellectual content: YK. Final approval of the version to be published: All authors. All authors read and approved the final manuscript.

\section{Funding}

Domestic support from (1) National Natural Science Foundation of China (No. 81772547); (2) the Fundamental Research Funds for the central Universities (No. 2017SCU04A18); (3) Young scientific and academic leaders training program of Sichuan University (No. 0082604151001/035); (4) Foundation of Science \& Technology Department of Sichuan Province (No. 2019YFS0256); (5) 1.3. 5 project for disciplines of excellence, West China Hospital, Sichuan University (No. ZY2017304).

\section{Availability of data and materials}

The datasets generated and/or analyzed during the study can be obtained from the corresponding Author on reasonable request.

\section{Declarations}

\section{Ethics approval and consent to participate}

The application of data from this database has been approved by the Biomedical Ethics Committee of the West China Hospital, Sichuan University, China [IRB No. 2014(215)]. Meanwhile, signed informed consent for this study was waived per the approval of Biomedical Ethics Committee of the West China Hospital, Sichuan University, since this was a retrospective study. And the data of patients were summarized and analyzed anonymously. All procedures performed in this study involving human participants were in accordance with the ethical standards of the institutional review board of ethics committee and national research committee with the 1964 Helsinki Declaration and its later amendments.

\section{Consent to publish}

Not applicable.

\section{Competing interests}

No competing interests.

\begin{abstract}
Author details
${ }^{1}$ Department of Gastrointestinal Surgery, West China Hospital, Sichuan University, No. 37 Guo Xue Xiang Street, Chengdu 610041, Sichuan Province, China. ${ }^{2}$ Institute of Gastric Cancer, State Key Laboratory of Biotherapy/Collaborative Innovation Center of Biotherapy and Cancer Center, West China Hospital, Sichuan University, Chengdu, China. ${ }^{3}$ Department of Gastrointestinal Surgery, West China Hospital Sichuan Univerity Jintang Hospital, Chengdu, China.
\end{abstract}

Received: 24 October 2020 Accepted: 5 April 2021 Published online: 26 April 2021

\section{References}

1. Siegel RL, Miller KD, Jemal A. Cancer statistics, 2019. CA Cancer J Clin. 2019:69:7-34.

2. Bray F, Ferlay J, Soerjomataram I, et al. Global cancer statistics 2018: GLOBOCAN estimates of incidence and mortality worldwide for 36 cancers in 185 countries. CA Cancer J Clin. 2018;68:394-424.

3. Chen W, Zheng R, Baade PD, et al. Cancer statistics in China, 2015. CA Cancer J Clin. 2016;66:115-32.

4. Colquhoun A, Arnold M, Ferlay J, et al. Global patterns of cardia and noncardia gastric cancer incidence in 2012. Gut. 2015;64:1881-8.

5. Liu K, Yang K, Zhang W, et al. Changes of esophagogastric junctional adenocarcinoma and gastroesophageal reflux disease among surgical patients during 1988-2012: a single-institution, high-volume experience in China. Ann Surg. 2016;263:88-95.

6. Japanese Gastric Cancer Association. Japanese gastric cancer treatment guidelines 2014 (ver. 4). Gastric Cancer 2017;20:1-19.

7. Songun I, Putter $H$, Kranenbarg EM, et al. Surgical treatment of gastric cancer: 15-year follow-up results of the randomised nationwide Dutch D1D2 trial. Lancet Oncol. 2010;11:439-49.

8. Japanese Gastric Cancer Association. Japanese classification of gastric carcinoma: 3rd English edition. Gastric Cancer 2011;14:101-112.

9. Liu CG, Lu P, Lu Y, et al. Distribution of solitary lymph nodes in primary gastric cancer: a retrospective study and clinical implications. World J Gastroenterol. 2007;13:4776-80.

10. Hu Y, Huang C, Sun Y, et al. Morbidity and mortality of laparoscopic versus open D2 distal gastrectomy for advanced gastric cancer: a randomized controlled trial. J Clin Oncol. 2016;34:1350-7.

11. Inaki N, Etoh T, Ohyama T, et al. A multi-institutional, prospective, phase II feasibility study of laparoscopy-assisted distal gastrectomy with D2 lymph node dissection for locally advanced gastric cancer (JLSSG0901). World J Surg. 2015;39:2734-41.

12. Kinoshita T, Uyama I, Terashima M, LOC-A Study Group, et al. Long-term Outcomes of Laparoscopic Versus Open Surgery for Clinical Stage I//II Gastric Cancer: A Multicenter Cohort Study in Japan (LOC-A Study). Ann Surg. 2019;269:887-94.

13. Park YK, Yoon HM, Kim YW, COACT group, et al. Laparoscopy-assisted versus open D2 distal gastrectomy for advanced gastric cancer: results from a randomized Phase II multicenter clinical trial (COACT 1001). Ann Surg. 2018;267:638-45.

14. Zhang $\mathrm{WH}$, Song $\mathrm{XH}$, Chen $X Z$, et al. Characteristics and survival outcomes related to the infra-pyloric lymph node status of gastric cancer patients. World J Surg Oncol. 2018;16:116.

15. Zhang WH, Yang $K$, Chen XZ, et al. Clockwise, modularized lymphadenectomy in laparoscopic gastric cancer surgery: a new laparoscopic surgery model. J Gastrointest Surg. 2019;23:895-903.

16. Section of Laparoscopic \& Endoscopic Surgery, Branch of Surgery, Chinese Medical Association; Robotic and Laparoscopic Surgery Committee of Chinese Research Hospital Association. [Guideline for laparoscopic gastrectomy for gastric cancer (2016 edition)]. Zhonghua Xiao Hua Wai Ke Za Zhi = Chinese Journal of Digestive Surgery 2016;15:851-857.

17. Chinese Society of Laparoscopic Surgery, Chinese Endoscopist Association, Chinese Medical Doctor Association; Robotic and Laparoscopic Surgery Committee of Chinese Research Hospital Association; Chinese Laparoscopic Gastrointestinal Surgery Study Group. [Expert consensus on quality control of the laparoscopic radical resection for gastric cancer in China (2017 edition)]. Zhonghua Xiao Hua Wai Ke Za Zhi = Chinese Journal of Digestive Surgery 2017;16:539-547.

18. Washington K. 7th edition of the AJCC cancer staging manual: stomach. Ann Surg Oncol. 2010;17:3077-9.

19. Chen QY, Huang CM, Lin JX, et al. Laparoscopic infrapyloric area lymph node dissection with no. $14 \mathrm{v}$ enlargement for advanced lower gastric cancer in middle colic vein approach. Ann Surg Oncol. 2016;23:951.

20. Son T, Lee JH, Kim YM, et al. Robotic spleen-preserving total gastrectomy for gastric cancer: comparison with conventional laparoscopic procedure. Surg Endosc. 2014;28:2606-15.

21. Shinohara H, Kurahashi Y, Kanaya S, et al. Topographic anatomy and laparoscopic technique for dissection of no. 6 infrapyloric lymph nodes in gastric cancer surgery. Gastric Cancer. 2013;16:615-20.

22. Shibasaki S, Suda K, Nakauchi M, et al. Outermost layer-oriented medial approach for infrapyloric nodal dissection in laparoscopic distal gastrectomy. Surg Endosc. 2018;32:2137-48. 
23. Cao LL, Huang CM, Lu J, et al. The impact of confluence types of the right gastroepiploic vein on no. 6 lymphadenectomy during laparoscopic radical gastrectomy. Medicine (Baltimore). 2015;94:e1383.

24. Yang $K$, Zhang WH, Liu K, et al. Comparison of quality of life between Billroth-I and Roux-en-Y anastomosis after distal gastrectomy for gastric cancer: a randomized controlled trial. Sci Rep. 2017;7:11245.

25. Zuo $\mathrm{CH}$, Xie H, Liu J, et al. Characterization of lymph node metastasis and its clinical significance in the surgical treatment of gastric cancer. Mol Clin Oncol. 2014:2:821-6.

26. HundahI SA. The potential value of bursectomy in operations for transserosal gastric adenocarcinoma. Gastric Cancer. 2012;15:3-4.

27. Blouhos K, Boulas KA, Tsalis K, et al. Right-sided bursectomy as an access plane for aesthetic resection of the posterior leaf of the lesser sac from the head of the pancreas en block with the No. 6 and 14v lymph nodes in advanced lower third gastric cancer. Surgery. 2015;158:1742.

28. Kurokawa Y, Doki Y, Mizusawa J, et al. Bursectomy versus omentectomy alone for resectable gastric cancer (JCOG1001): a phase 3, open-label, randomised controlled trial. Lancet Gastroenterol Hepatol. 2018;3:460-8.
29. Zhang WH, Chen XZ, Yang K, et al. Bursectomy and non-bursectomy D2 gastrectomy for advanced gastric cancer, initial experience from a single institution in China. World J Surg Oncol. 2015;13:332.

30. Marutsuka T, Shimada S, Shiomori K, et al. Mechanisms of peritoneal metastasis after operation for non-serosa-invasive gastric carcinoma: an ultrarapid detection system for intraperitoneal free cancer cells and a prophylactic strategy for peritoneal metastasis. Clin Cancer Res. 2003;9:678-85

31. Han TS, Kong SH, Lee HJ, et al. Dissemination of free cancer cells from the gastric lumen and from perigastric lymphovascular pedicles during radical gastric cancer surgery. Ann Surg Oncol. 2011;18:2818-25.

\section{Publisher's Note}

Springer Nature remains neutral with regard to jurisdictional claims in published maps and institutional affiliations.
Ready to submit your research? Choose BMC and benefit from:

- fast, convenient online submission

- thorough peer review by experienced researchers in your field

- rapid publication on acceptance

- support for research data, including large and complex data types

- gold Open Access which fosters wider collaboration and increased citations

- maximum visibility for your research: over $100 \mathrm{M}$ website views per year

At BMC, research is always in progress.

Learn more biomedcentral.com/submissions 